Conclusions: The rheumatoid foot alters function. It causes disability which is related to foot pain, rearfoot podiatric abnormalities and disease activity. Disclosure of Interest: None declared

DOI: 10.1136/annrheumdis-2017-eular.5508

\section{AB0253 TIPS ON SELECTION OF BIOLOGICS FOR PATIENTS WITH RHEUMATOID ARTHRITIS BASED ON TREATMENT PATTERNS}

M. Kamiya ${ }^{1}$, S. Soen ${ }^{1}$, H. Kikuchi ${ }^{2}$, N. Okada ${ }^{3} .{ }^{1}$ Department of Orthopaedic Surgery and Rheumatology, Kindai University Nara Hospital, Ikoma-City;

${ }^{2}$ Department of Orthopaedic Surgery and Rheumatology, Kindai University Sakai Hospital, Sakai-City; ${ }^{3}$ Department of Orthopaedic Surgery, Sumoto Itsuki Hospital, Sumoto-City, Japan

Background: The emergence of biologics has led to innovation in the treatment of rheumatoid arthritis (RA). In the clinical setting, biologics are administered with careful consideration of complications and medical history in accordance with the treat-to-target recommendations. However, the progression of joint damage, the costs incurred before finding an effective biologic are serious concerns. It is therefore desirable to use biologics with long-term efficacy and less financial burden from the early stage.

Objectives: Participants were RA patients treated with one of three biologics having different mechanisms of action who achieved therapy targets with longterm treatment efficacy and consequently achieved either reduction or withdrawal of therapy. Patients' background characteristics and long-term treatment patterns were evaluated.

Methods: Between November 2004 and October 2016, 196, 57, and 85 RA patients were treated with etanercept (ETN), tocilizumab (TCZ), and abatacept $(A B T)$, respectively, in first- or second-line therapy. These patients were divided into the continuation group, who underwent therapy with the same agent for $>3$ years without disease flare (DAS28-ESR >3.2) persisting 3 months, and the discontinuation group, who experienced primary failure resulting in discontinuation of the therapy within 3 months. Student's t test or Mann-Whitney's U test were used to compare patients' background characteristics between the two groups for each biologic. Further, log-rank test and Steel-Dwass test, respectively, were used to compare therapy continuation rates and reasons for discontinuation among the three biologics. Finally, relative dose intensity (RDI) was calculated to evaluate the treatment patterns of the individual biologics.

Results: The Kaplan-Meier method showed that the 3-year continuation rates of therapy with ETN, TCZ, and ABT were $54.2 \%, 23.8 \%$, and $35.8 \%$, respectively: the continuation rate of ETN was significantly higher than that of the other two agents. The numbers of patients treated with ETN, TCZ, and ABT were respectively 46,9 , and 14 in the continuation group and 16, 12, and 11 in the discontinuation group. The proportion of patients treated with ETN plus concomitant MTX was significantly higher in the continuation group than in the discontinuation group $(\mathrm{P}=0.0057)$. No significant differences were found in patients' background characteristics (disease duration, rheumatoid or anti-cyclic citrullinated peptide positivity, number of biologics previously used, and DAS28ESR). Mean RDI values (median value, 95\% confidence interval) over a 3-year period were as follows: $0.95(0.92,0.83-1.06)$ for $25 \mathrm{mg} /$ week ETN therapy; 0.78 $(0.90,0.66-0.89)$ for $50 \mathrm{mg} /$ week ETN therapy; $0.84(0.84,0.76-0.89)$ for TCZ therapy; and $0.87(0.94,0.79-0.95)$ for ABT therapy. The cumulative costs for 3 years of the respective treatments were 19,700,32,200, 27,300, and 39,000 euros ( 1 euro $=115$ Japanese yen). After targets were reached, the dose of ETN was maintained at $25 \mathrm{mg} /$ week or reduced from $50 \mathrm{mg} /$ week, while the TCZ and ABT therapies were continued over the long term with a longer dosing interval. Conclusions: Treatment with ETN plus concomitant MTX showed high continuation rates, and long-term achievement of therapy targets was maintained at a lower dosage (and thus, lower costs). It is beneficial to choose this method over non-TNF inhibitors.

Disclosure of Interest: None declared

DOI: 10.1136/annrheumdis-2017-eular.1146

\section{AB0254 COMPARISON OF DYNAMIC PEDOBAROGRAPHIC FINDINGS BETWEEN RHEUMATOID ARTHRITIS PATIENTS AND HEALTHY INDIVIDUALS}

S. Ghadimy, M. Aarabi, N. Abdolahi, N. Nematollahi, M. Aghaei. Rheumatology Reasearch Center, Gorgan, Iran, Islamic Republic Of

Background: Foot involvement happens early in RA patients and situation becomes ugly and painful rather very fast. There is a blank gap between starting of foot involvement and the time where patient needs surgical intervention for correcting the damages done by RA. No guideline exists for determining when and what we can do in these patients to detect and prevent the changes or at least reduce their damages.(1)

Objectives: 1. Evaluating foot pressure changes by pedobaroghy:

- comparing maximum force/peak pressure distributed in different regions of foot between RA patients and healthy individuals.

- comparing maximum force/peak pressure distributed in different regions of foot between men and women.

- determining effects of aging on maximum force/peak pressureby comparing them between two age groups.
- determining effects of duration of RA on force/peak pressure in RA patients.

2. Evaluating radiologic findings in symptomatic RA patients and comparing them with pedobarographic findings.

3. Evaluating and comparing quality of life in RA patients and healthy individuals with SF36 questionnaire.

Methods: 90 RA patients and 45 healthy individuals were chosen and entered this research. Patients divided into two groups: 45 patients without any previous foot symptoms, 45 with foot symptoms. All these groups underwent dynamic pedobarography with Novel emed pedobargraphy.

We took foot and ankle X-rays for symptomatic group.

Based on Larsen score, symptomatic patients were divided into individuals with and without radiologic changes.

Results: There was a significant difference in total maximum force between patients and healthy individuals; healthy individuals had lesser amount of maximum force in different parts of their feet compared to RA patients.

Fore foot region endured the most amount of maximum force and pressure in all three groups with no significant difference between groups.

RA patients both symptomatic and asymptomatic had more force and pressure upon their midfoot regions compared to the healthy group p-value:.000.

Since we excluded patients with severe deformities and those who couldn't walk alone from the study, and our cases were relatively in early stages of disease, we didn't find any significant difference in pressure or force between symptomatic patients with and without radiographic findings. This can be explained by the fact that foot pressure alteration detectable in pedobarography is already begun in all patients with foot symptoms but radiologically evident pathologies had not happened yet.

Conclusions: RA has considerable effect on patients' feet along with other physical and mental issues. While conventional radiologic methods has a limited eficacy in predicting and diagnosing the pathologic changes in foot region, pedobarography can easily shows these changes in foot pressure values and can be used to detect RA patients that need simple interventions like using proper insoles to prevent surgical interventions

References:

[1] Schmiegel A, Rosenbaum D, Schorat A, Hilker A, Gaubitz M. Assessment of foot impairment in rheumatoid arthritis patients by dynamic pedobarography. Gait \& posture. 2008;27(1):110-4.

Disclosure of Interest: None declared

DOI: 10.1136/annrheumdis-2017-eular.4445

\section{AB0255 EVALUATION OF KINESIOPHOBIA IN PATIENTS WITH RHEUMATOID ARTHRITIS AND ANKYLOSING SPONDYLITIS}

M. Aykut ${ }^{1}$, B. Cakit ${ }^{2}$, E. Mert ${ }^{2}$, S. Aslan ${ }^{2}$, E. Sahingoz ${ }^{2}$, H. Genc ${ }^{2}$, F.F. Ayhan ${ }^{2}$, A. Karagoz ${ }^{2}$. ${ }^{1}$ Physical Medicine and Rehabilitation, Ankara Training and Research Hospital; ${ }^{2}$ Physical Medicine and Rehabilitation, Ankara Training and Research Hospital, Ankara, Turkey

Background: Fear avoidance behavior which is caused by painful injury resulting precision and extreme fear is defined as kinesiophobia. Rheumatoid arthritis (RA) is a chronic, inflammatory and systemic disease with symmetrical arthritis and visceral involvement. Ankylosing spondylitis (AS) is a chronic, inflammatory disease with involvement of the spine or peripheral joints.

Objectives: In our study, we aimed to evaluate the relationships between kinesophobia and disease activity, quality of life (QoL), level of physical activity and emotional status in RA and AS patients.

Methods: We included 42 patients with RA (8 males-M, 34 females-F) (group 1), 49 patients with AS (34 M, $15 \mathrm{~F}$ ) (group 2) and 29 healthy controls (9 M, $20 \mathrm{~F}$ ) (group 3) in our study. The QoL was assessed using the health assessment questionnaire $(\mathrm{HAQ})$, kinesiophobia was assesed with Tampa scale of kinesiophobia (TSK), pain was assesed with visual analog scale (VAS), fatigue was assesed with VAS and emotional status was assesed with Beck depression inventory (BDI). Disease activity was assesed with Bath ankylosing spondylitis disease acitivity index (BASDAI) and functional status was assesed with Bath ankylosing spondylitis functional index (BASFI) in patients with AS. Disease activity was assesed with DAS28 in patients with RA.

Results: The mean age was 46.2 in group 1, 43.2 in group 2 and 40.17 in group 3 . There was no difference among groups with respect to mean age $(p>0.05)$. Kinesiophobia was present in 37 patients in group 1, 22 patients in group 2 and 7 patients in group 3. Statistically significant differences were found among groups

Table 1. Baseline features of the patients of AS and RA and healthy controls

\begin{tabular}{lccc}
\hline & Group 1 & Group 2 & Group 3 \\
\hline Age & $46,2 \pm 11,47$ & $43,2 \pm 10,73$ & $40,17 \pm 7,77$ \\
Gender (F/M) & $34 / 8$ & $15 / 34$ & $20 / 9$ \\
VAS $^{*}$ & $47,02 \pm 24,42$ & $32,44 \pm 26,75$ & $1,72 \pm 4,68$ \\
TKS $^{* *}$ & $44,73 \pm 7,26$ & $36 \pm 12,03$ & $29,58 \pm 9,37$ \\
Fatigue $^{*}(\mathrm{VAS})^{\dagger}$ & $55,47 \pm 24,31$ & $36,93 \pm 27,70$ & $37,93 \pm 20,59$ \\
$\mathrm{HAQ}^{\ddagger}$ & $0,73 \pm 0,83$ & $0,43 \pm 0,41$ & $0,06 \pm 0,19$ \\
$\mathrm{BDI}^{\S}$ & $14,17 \pm 9,49$ & $12,23 \pm 9,63$ & $5,25 \pm 6,13$ \\
\hline${ }^{*} \mathrm{p}=0.008$ bet
\end{tabular}

group 1 and $2 ; p<0.001$ between group 2 and $3 ; p<0.001$ between group 1 and $3 .{ }^{* *} p<0.001$ between group 1 and 2; $p=0.023$ between group 2 and 3; $p<0.001$ between group 1 and $3 .{ }^{\dagger} p<0,05$ between group 1 and $2 ; 2$ and $3 ; 1$ and $3 .{ }^{\ddagger} p=0.039$ between group 1 and $2 ; p=0.021$ betwen group 2 and $3 ; p<0.001$ between group 1 and $3 .{ }^{\S} p>0.05$ between group 1 and $2 ; p=0.004$ betwen group 2 and $3 ; p<0.001$ between group 1 and 3 . 